\title{
Developments in a growing field
}

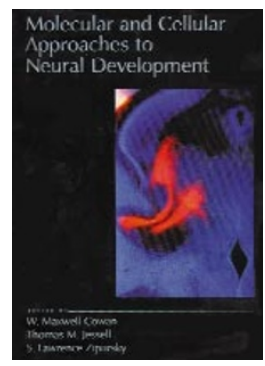

\section{Molecular and Cellular Approaches to Neural Development}

Edited by W. M. Cowan, T. M. Jessell and S. L. Zipursky

Oxford University Press, 1997. \$65 hardcover, pp 563

ISBN 0-19-511166-4

Reviewed by Roger Keynes

Our understanding of neural development has come a long way since the heyday of descriptive analysis a century ago, famously exemplified by Cajal's ability to see function and mechanism down the microscope. By the 1930s a more experimental emphasis had taken over, culminating in the discovery by Spemann and Mangold of neural induction. This promised insight into not only the patterning of the vertebrate embryo and its nervous system, but also the nature of molecular instruction itself. The field duly adopted a biochemical approach, but identifying the inducers proved to be beyond the techniques of the day. By the mid1950s, in the main developmental biology textbook of the time, Paul Weiss was driven to conclude his chapter on neural development with the remark that "The only valid summary...is to say that, by the very nature of the developmental process, it does not lend itself to verbal summarization". A glance at his ensuing diagram also suggested that it did not lend itself to visual summarization any more easily.

As will be clear from this book, the position has been transformed by progress in the field during the past 20 years, and it is no longer enough (if indeed it ever was) to teach students the dry bones of description. There are probably several reasons for this, the most obvious being the pace of technical advances in cell and molecular biology. For an aspiring neurobiologist, working out how a particular piece of the nervous system develops may seem more tractable than studying how it works or what it does, and this may have attracted increasing numbers into development in the 1980s. Simultaneously, the complexity of the developing nervous system has made it a good hunting ground

Roger Keynes is at the Department of Anatomy, University of Cambridge, Downing Street, Cambridge CB2 3DY, UK

email: rjk10@cus.cam.ac.uk for novel gene expression patterns and mutant phenotypes, and this is likely to continue as more genes come through from large-scale sequencing projects.

Despite the rapid rate of progress over the past few years, there has been a dearth of comprehensive accounts of the individual success stories gathered into a single volume. This multi-author book goes a long way in filling the gap. It arose from a meeting held at the Howard Hughes Medical Institute in 1996, and the contributors, all leaders in the field, have done more than just review their own research. A particular feature is the collaborative nature of most of the chapters, which cover a common problem using different animal systems, or which bring together those with overlapping interests. With the stated aim of increasing perspective, this has worked well, and the editors are to be congratulated. The conserved elements of development in different organisms, each with particular experimental advantages, are well brought out, but so also are the differences.

Not surprisingly, the emphasis is on the remarkable advances at the cellular and molecular levels. Most key areas where progress has been especially impressive are represented, and individual chapters range from neural specification and patterning, through axon guidance and synapse formation, to the details of the development of the fly's eye and the mammalian cerebral cortex. The reader is informed, for example, how known inducers of amphibian nerve cells block a molecular signalling pathway that would otherwise make them epidermal, how the four main classes of axon guidance cue are mechanistically related and phylogenetically conserved, and how multifarious are the actions of the neurotrophins.

The coverage will suit the already-initiated better than those requiring an introduction, and is ideal for graduate students and others casting for the broad view. In the wake of the rich pickings of the last few years, it is worth asking whether the researcher entering the field is given some idea of what remains to be done. A number of the contributors comment on our ignorance about the overall 'logic' of neural development despite all these advances, but this is perhaps too broad to be helpful. Several attempts are made, however, to delineate outstanding questions more specifically. How, for example, is the mammalian nervous system induced, and how are the signals controlling the early stages of neurogenesis linked to those that actually build the neuron? To what extent does the neuromuscular junction model synaptogenesis in the CNS? How do distinct nuclei appear in the thalamus, and how do different neuronal subtypes appear at similar positions in the neural tube? What are the molecular mechanisms that guide axon projections to and from the cerebral cortex, and what are the layer-specific molecular cues that determine vertical cortical circuitry? As one contributor points out, the scale of the problems of neural development can make it difficult to know where to start, but framing such questions should be useful.

Another, perennial question is whether a detailed understanding of the development of the nervous system will help us to understand how it works. A robust view appears in the chapter by Mario Capecchi, which states that "virtually as a rule in biology, it has been found that the function of a system, whether we are speaking of individual molecules or of complex systems, is derived from its structure. The structure in turn is derived intrinsically from its synthesis. If we understand in detailed molecular terms how the hindbrain is made, we may well be a long way along the road to understanding how it functions." Some may disagree, given the disparity between what is already known about connectivity and function in areas such as the cerebellum. The similarities in the construction of neurons compared with other cells may be more impressive than the differences, given present knowledge, but it could be argued that the reverse is true for the function of neuronal assemblies. It should suffice nevertheless, and regardless of the attendant potential for solving difficult clinical problems, that the development of the nervous system is fascinating in its own right, as amply demonstrated in this excellent book.

For the full table of contents see

http://www.hhmi.org/home/research/neural.html 